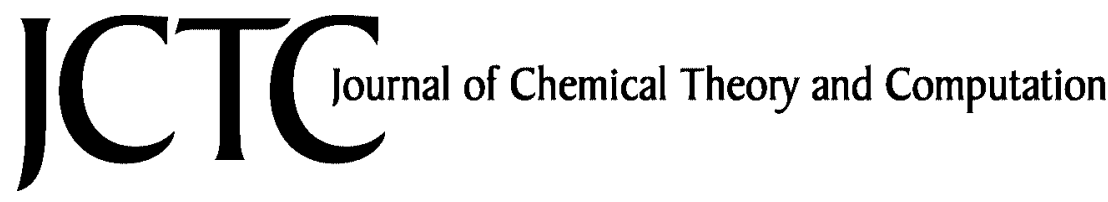

\section{Fragment-Localized Kohn-Sham Orbitals via a Singles Configuration-Interaction Procedure and Application to Local Properties and Intermolecular Energy Decomposition Analysis ${ }^{\dagger}$}

\author{
Peter Reinhardt,* Jean-Philip Piquemal, and Andreas Savin \\ UPMC University Paris 06, Laboratoire de Chimie Théorique, 4 place Jussieu, \\ F-75252 Paris, France, and CNRS UMR 7616, Laboratoire de Chimie Théorique, \\ 4 place Jussieu, F-75252 Paris, France
}

Received June 24, 2008

\begin{abstract}
As for generating localized Hartree-Fock orbitals, we propose a potentially linearscaling singles-Cl scheme to construct fragment-localized density functional theory (DFT) orbitals for molecular systems as water clusters. Due to the use of a deformation step instead of a localization step, the influence of the environment on each separate molecule can be studied in detail. The generated orbital set for the whole molecular system is strictly equivalent to a set of canonical orbitals and is a subsequent energy decomposition of intermolecular interactions into electrostatic, exchange repulsion, and orbital interaction, well beyond dimer systems. Beyond this, the correspondence of the individual orbitals to the initial monomer orbitals permits to assess how an interaction deforms an electron density. We show this for dipole moments, which may be decomposed into monomer contributions, polarization, and charge-transfer contribution. Applications to a water and an ammonia dimer and chains of water molecules show possible further developments toward multipolar expansions and other orbital-based schemes for parametrizing force fields.
\end{abstract}

\section{Introduction}

Density functional theory (DFT) is now a widely used tool for calculating molecular properties due to a favorable scaling with system size, permitting calculations at high precision even for medium-sized molecules, inaccessible to perturbation- or configuration-interaction (CI) based quantum chemical methods.

Another advantage of DFT lies in the fact that a single Hartree product or Slater determinant with Kohn-Sham orbitals is usually employed for describing the wave function and not a long expansion in reference and excited determinants. The connection between the density and orbitals is therefore straightforward as in Hartree-Fock, nevertheless including correlation through the functional.

\footnotetext{
${ }^{\dagger}$ Article honoring the work of Jean-Pierre Daudey (Toulouse, France).

* E-mail: Peter.Reinhardt@upmc.fr.
}

This Kohn-Sham wave function-the orbitals generating the density, assembled in a Hartree product or in a Slater determinant-is invariant under unitary transformations of the occupied orbitals. This property, although used, may deserve more attention for molecular systems, as it is a recurrent topic in modern wave function-based quantum chemistry, notably in the quest for linear-scaling algorithms. ${ }^{1}$

Orbital localization has a longstanding tradition in quantum chemistry (see ref 2 and references therein) and is associated with the names of Boys, ${ }^{3}$ Edmiston-Ruedenberg, ${ }^{4}$ PipekMezey, ${ }^{5}$ or even the natural bond orbital (NBO) analysis of Weinhold et al. ${ }^{6,7}$ The Boys localization is known in the physicists community as Marzari-Vanderbilt ${ }^{8}$ scheme. These methods optimize a localization criterion minimizing bielectronic repulsion (Boys) or the fragmentation of the orbitals on atomic centers (Pipek-Mezey). Commonly the starting point for these localization methods is a set of completely delocalized canonical orbitals, diagonalizing the Fock or Kohn-Sham matrix. The use of delocalized orbitals as 
starting point becomes rapidly a handicap for larger systems, motivating the development of intrinsic localization procedures without the necessity to generate completely delocalized orbitals.

Recently, several approaches have been proposed in order to use localization criteria to unravel the origin of intermolecular interactions in weakly interacting molecular systems. For example, Rob et al. ${ }^{9}$ presented a approach based on Boys' localization for the computation of intermolecular electrostatic energies including correlation showing promising results which could be competitive with density fitting approaches ${ }^{10,11}$ as cutoff approximations lead to errors below $0.5 \mathrm{kcal} / \mathrm{mol}$ per dimer interaction. At the same time, a full energy decomposition analysis (EDA) was achieved by Khaliullin et al. ${ }^{12,13}$ using "absolutely localized orbitals" demonstrating the advantage of the localization process for computations on large systems. Such results are important as EDA are essential to the development and the calibration of advanced force fields. ${ }^{11,14}$

For constructing intrinsically localized DFT molecular orbitals, we take up an old idea, ${ }^{15}$ further developed and published by Daudey ${ }^{16}$ and still cited recently, ${ }^{2,17,18}$ without the need to have delocalized canonical orbitals. The central point is that in Hartree-Fock theory a CI of singly excited Slater determinants lowers the total energy toward a set of Hartree-Fock orbitals, reaching convergence when satisfying Brillouin's theorem: interactions between occupied and virtual orbitals via the Fock matrix vanish. The singles-CI matrix is locally concentrated, as excitations on fragments with large spatial separation are much less important than those within close neighborhood. That permits the local correction of the monomer orbitals, disregarding the actual extent of the complete system. No canonical (completely delocalized) molecular orbitals are created or needed.

The method becomes operational and potentially linear scaling when taking for the elements of the configuration interaction matrix between singly excited determinants only the part generated from Fock matrix elements as

$$
\left\langle\Phi_{i}^{a}|\mathbf{H}| \Phi_{j}^{b}\right\rangle \approx F_{a b} \delta_{i j}-F_{i j} \delta_{a b}
$$

Otherwise a partial four-index transformation would be necessary for each SCF iteration. ${ }^{19}$

The use of density-functional theory for intermolecular interactions may seem inappropriate, as the important dispersion part is not correctly treated in actual Kohn-Sham formalism. However, improvements are under way, ${ }^{20}$ and the scheme we like to present here will be directly applicable to these improved functionals as well. On the other hand, we may cite several successful DFT applications for hydrogenbonded systems, ${ }^{21}$ despite this inherent default.

We should stress again that the application of the present orbital localization scheme does not help to overcome basic defaults of current density functional theory by inclusion of missing parts of the correlation energy. Even though we employ a CI-based optimization scheme, nothing is for the moment included to go beyond a common Kohn-Sham energy, obtainable via standard procedures. Nevertheless, the generated local orbitals and moments developed here may be helpful in a subsequent step to develop a proper dispersion scheme like in the recent DFT based SAPT (symmetry adapted perturbation theory), where the Kohn-Sham monomer orbitals act as starting point for a perturbative intermolecular scheme. ${ }^{22,23}$

In the SCF MI approach (SCF for molecular interaction) of Khaliullin et al., ${ }^{12,13}$ a similar scheme for orbital optimization is presented, replacing the iterative singles-CI step by one single calculation of fourth-order perturbation theory in monomer orbitals without explicit orthogonalization of the complete orbital set.

For the sake of completeness, we may cite the work of Adams and Gilbert with a similar objective, ${ }^{24,25}$ constructing local fragment orbitals in the sense of building blocks for a molecule or solid, further developed by $\mathrm{Kunz}^{26}$ and Seijo and Bandiaran. ${ }^{27}$

The paper is further organized as follows: in the first part, we recall the iteration scheme based of the CI step and our particular use for treating weakly interacting multimonomer or segmented systems. We show how the procedure allows the decomposition of any monoelectronic property into welldefined monomer contributions and how the scheme fits into common energy decomposition schemes like those of Ziegler and Rauk, ${ }^{28}$ Bickelhaupt and Baerends, ${ }^{29}$ or the constrained space orbital variations approach (CSOV) of Bagus et al. ${ }^{30}$

In the second part, results are shown for the dipole moments (local and global) of an ammonia dimer, the energy decomposition for a linear water dimer, ${ }^{31,32}$ and an orbital analysis and an energy decomposition for small, helixlike water clusters (denoted longitudinal hydrogen-bonded chains (l-HBCs), see ref 33 and the Appendix) in order to identify the convergence and transferability of the orbital decomposition.

In the Appendix, we collect all technical details and the formal proof of the equivalence of the singles-CI scheme for obtaining Hartree-Fock or Kohn-Sham orbitals.

\section{Methodological Details}

We consider a system which may be decomposed intuitively into well-separated fragments. For each fragment, we construct canonical orbitals separately, in a set of atomic orbitals attached only to the particular fragment. From this calculation, we keep the virtual orbitals for spanning the virtual orbital space of the complete system. To obtain reasonable occupied orbitals of the monomers, we perform in a next step a calculation of canonical orbitals of the individual monomers in the atomic-orbital basis of the complete system. The advantage of such a procedure lies in the fact that the necessary integrals over atomic orbitals have to be calculated only once, and all iterations can be performed with the same set of integrals. At the end of the double series of monomer calculations, the orbitals are assembled to a starting set of occupied orbitals and orthogonalized employing Löwdin's $\mathbf{S}^{-1 / 2}$ orthogonalization. ${ }^{34}$ This "democratic" orthogonalization insures that on average every orbital is corrected in a least-most perturbation, preserving as most as possible the characteristics of the monomer orbitals. ${ }^{35}$ To this set of occupied starting orbitals, the previously generated set of virtual orbitals is orthogonalized through projection, and at last, the virtual orbitals are orthogonalized among themselves, again via Löwdin's procedure. 
2.1. SCF Iterations through a Singles-CI. Now everything is set up for starting the SCF procedure toward the HF or DFT wave function of the whole system. A first determinant $\Phi_{0}$ is constructed from the occupied starting orbitals, and the energy is lowered by variational inclusion of monoexcited determinants $\Phi_{i}^{a}$

$$
\Psi=\Phi_{0}+\sum_{i, a} c_{i}^{a} \Phi_{i}^{a}
$$

from which we take the coefficients to correct in first-order the occupied and virtual orbitals $\phi_{i}(\vec{r})$ and $\phi_{a}(\vec{r})$, respectively, through

$$
\begin{aligned}
& \phi_{i}^{\prime}(\vec{r})=\phi_{i}(\vec{r})+\sum_{a} c_{i}^{a} \phi_{a}(\vec{r}) \\
& \phi_{a}^{\prime}(\vec{r})=\phi_{a}(\vec{r})+\sum_{i} c_{i}^{a} \phi_{i}(\vec{r})
\end{aligned}
$$

The correction of the virtual orbitals ensures that the occupied and virtual space remain well orthogonal with respect to each other. However, orthogonality within each of the two spaces is only ensured to first order, and the orbitals are orthogonalized again using Löwdin's procedure. With the orthogonal orbitals at hand, we construct again the singles-CI matrix, look for its lowest eigenvalue and corresponding eigenvector, and correct the orbitals, etc. The loop is controlled either by the changing of the total energy or by the smallness of the mean Fock (or Kohn-Sham) matrix element $\mathbf{F}_{i a}$, coupling occupied and virtual orbitals. If one insists (and making useless the effort of preserving locality), the generated (localized) orbital set can be transformed directly to a canonical one by a single diagonalization of the Fock (or the Kohn-Sham matrix in the present case). The final determinant (Hartree-Fock or Kohn-Sham) is indeed the lowest in energy as the energy evaluation is independent of the CI procedure, using in the Hartree-Fock case the Rayleigh quotient $\left\langle\Phi_{0}|\mathbf{H}| \Phi_{0}\right\rangle\left\langle\left\langle\Phi_{0} \mid \Phi_{0}\right\rangle\right.$ or, respectively, in the Kohn-Sham case, the corresponding energy functional $E[\rho]$ with a particular exchange-correlation part $E_{\mathrm{XC}}[\rho]$.

The cost of this CI procedure is equivalent to a straightforward iteration using a diagonalization of the Fock (or Kohn-Sham) matrix due to the use of an approximate CI matrix (eq 1) without an explicit four-index transformation of the bielectronic integrals at each iteration.

Linear scaling may be achieved because the orbital corrections due to the orthogonalization procedure and the Fock matrix elements are strongly localized in real space in the beginning and remain localized as unnecessary delocalizations due to any diagonalization are avoided.

As for all CI schemes, the question of size-consistency may be posed. Algorithms for the correction are well-known (Davidson correction, CEPA-like treatments, ${ }^{36}$ etc.) but seem not to be of crucial importance in the present, medium-sized cases. ${ }^{37}$ The final orbitals are the same, and only small improvements of convergence were observed.

2.2. Use of the Orbital Deformations for Analyzing the Wave Functions. As we have at the end orbitals associated to the monomer orbitals, we may try to expand an according density for example in multipolar moments or consider just the deformation in a linear decomposition of the form

$$
\phi_{i}(\vec{r})=\phi_{i}^{\mathrm{M}}(\vec{r})+\phi_{i}^{\mathrm{S}}(\vec{r})+\phi_{i}^{\mathrm{O}}(\vec{r})
$$

where the subscripts stand for "monomer", "same monomer" and "other monomers" to describe the effect of on-site polarization and of charge transfer. The monomer part is the projection of $\phi_{i}(\vec{r})$ on the corresponding, unperturbed monomer orbital $\phi_{i}^{0}(\vec{r})$, the second part is the expansion of the remainder on the basis functions on the same monomer, and the third one the expansion on the basis functions located elsewhere.

The three parts are not orthogonal but give rise to six different density matrices

$$
P^{\mathrm{MM}, i}+P^{\mathrm{MS}, i}+P^{\mathrm{MO}, i}+P^{\mathrm{SS}, i}+P^{\mathrm{SO}, i}+P^{\mathrm{OO}, i}
$$

the sum of which is the partial density matrix created by the molecular orbital $\phi_{i}(\vec{r})$.

$$
P^{i}=\left|\phi_{i}\right\rangle\left\langle\phi_{i}\right|
$$

In the spirit of a Mulliken analysis, we may attribute densities between different parts in halves to the proper parts MM, SS, and OO as The analysis may be done for each

$$
\begin{aligned}
P^{i}= & \underbrace{P^{M M, i}+\frac{1}{2}\left(P^{M S, i}+P^{M O, i}\right)}_{\text {monomer }}+\underbrace{P^{S S, i}+\frac{1}{2}\left(P^{M S, i}+P^{S O, i}\right)}_{\text {polarization }}+ \\
& +\underbrace{P^{O O, i}+\frac{1}{2}\left(P^{M O, i}+P^{S O, i}\right)}_{\text {charge transfer }}
\end{aligned}
$$

orbital separately, showing thus more or less deformed orbitals of the cluster with respect to the individual monomers. Of course, this detailed decomposition depends on the form of the monomer orbitals and of that of the orbitals of the whole system. Nevertheless, the choice of canonical monomer orbitals and CI-generated multimer orbitals reduces this general ambiguity toward reproducible quantities.

$$
P^{i}=P_{\text {Mono }}^{i}+P_{\text {Polarization }}^{i}+P_{\mathrm{CT}}^{i}
$$

The sum of all of these partial density matrices gives the density matrix of the whole system.

$$
P^{\text {system }}=\sum_{i} P^{i}
$$

On the other hand, summing these orbital-specific density matrices over the occupied orbitals of each monomer separately,

$$
P^{\mathrm{frag}}=\sum_{i \in \mathrm{frag}} P^{i}
$$

we may decompose any monoelectronic operator into mono, polarization, and charge-transfer parts attributed to the individual monomers. We show this for the Mulliken populations and dipole moments in the next section. For instance with the dipole operator $\mu$, we have

$$
\begin{aligned}
\vec{\mu}^{\mathrm{frag}} & =P^{\mathrm{frag}} \vec{\mu}=\left(P_{\text {Mono }}^{\mathrm{frag}}+P_{\text {Polarization }}^{\mathrm{frag}}+P_{\mathrm{CT}}^{\mathrm{frag}}\right) \vec{\mu} \\
& =\vec{\mu}_{\text {Mono }}^{\mathrm{frag}}+\vec{\mu}_{\text {Polarization }}^{\mathrm{frag}}+\vec{\mu}_{\mathrm{CT}}^{\mathrm{frag}}
\end{aligned}
$$

Of course we have to remind that this decomposition is not unique, as it is based on orbitals. Nevertheless, starting from canonical monomer orbitals and employing the de- 
scribed singles-CI procedure gives a recipe to construct decompositions with a well-defined procedure. Our dipole moments sum up to the global dipole moment of the whole system under study, and following them individually when assembling a system may help to identify characteristic regions of the fragments.

The same principle may serve for total energy decompositions, the (Kohn-Sham) kinetic energy and the electron-nuclear attraction also being monoelectronic properties. However, the bielectronic terms (electron-electron Coulomb repulsion and Hartree-Fock exchange as for the B3LYP functional) become very numerous, and the attribution of their crossterms to individual monomers seems less reasonable than for the monoelectronic quantities. Therefore, we follow for the moment another proposition, as detailed below.

2.3. Intermolecular Energy Analysis. Intermolecular energy decompositions are often in the center of interest for singling out active sites of fragments and preponderant, chemically intuitive contributions to the overall stability of an assembly. One of the first attempts with great success was Morokuma's analysis of a Hartree-Fock interaction energy, ${ }^{38,39}$ using parts of the Fock matrix in the selfconsistent scheme in order to extract electrostatic, polarization, charge-transfer, and a sum of remaining terms. Instead of performing several independent calculations for obtaining the different contributions, we have all at hand for constructing the interaction energy step by step from the unrelaxed monomer orbitals (electrostatic interactions), the orthogonalized monomer orbitals (Pauli repulsion), and orbital interaction as relaxation of the orbitals from the starting orbitals of the singles CI step to the Kohn-Sham orbitals of the whole system. This scheme, initially proposed by Ziegler and Rauk, ${ }^{28}$ has been popularized by Bickelhaupt and Baerends ${ }^{29}$ and served recently for a comparison with the ab initio SAPT scheme. ${ }^{40}$ The CSOV decomposition scheme, in its spirit closer to Morokuma's original scheme as subsequently more and more orbitals are allowed to relax in harmony, gives as well results close to those of the Bickelhaupt and Baerends decomposition. ${ }^{41}$

As these energy decompositions are completely invariant under orbital localization, we should obtain exactly the same results as for canonical orbitals, unless employing approximations within the orbital space as cutoffs or selection schemes. The advantage of the use of (fragment-)localized orbitals instead of canonical ones lies in the fact that approximations become distance dependent and can be chosen prior to a calculation (to be carried out more efficiently) without loosing the necessary precision.

Beyond a common decomposition of one single dimer interaction (like in SAPT or CSOV), we may decompose the $n$-fragment interactions in larger clusters without any additional effort. Such multifragment decompositions at the DFT level have only to be carried out at the moment by Khaliullin and Head-Gordon ${ }^{12,13}$ and should be useful for obtaining reference data for force fields.

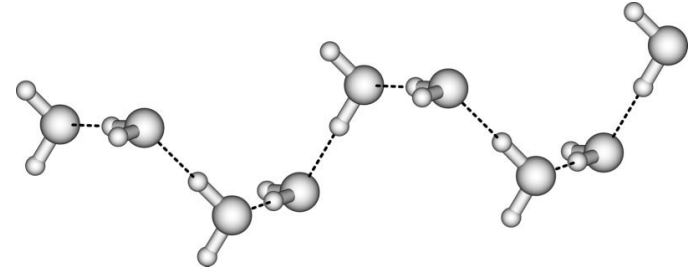

Figure 1. Water molecules in a helixlike linear structure. Pieces of one to five molecules from the left end of the chain have been considered in the present work.

\section{Applications}

The present approach has been applied to an ammonia dimer and a series of small water clusters. All technical details may be found in the Appendix.

The water cluster are parts of a helixlike arrangement (as depicted in Figure 1) of which we took pieces with two to five molecules.

3.1. Uniqueness of the Orbitals. The spread of the aforementioned decomposition (eq 7) gives us the possibility to measure the uniqueness of the generated orbitals for different conditions of their calculation. One can invoke the arbitrariness of the final orbitals to discredit the proposed generation scheme via the singles-CI step, as the final orbitals depend on the initial set of guess orbitals. Using canonical monomer orbitals as guess, this arbitrariness is already removed.

Here, we show that even different ways to achieve convergence, both through a constant Fock-matrix (or Kohn-Sham-matrix) mixing between the SCF cycles and a fixed scaling of the CI-vector, results only in minor differences for the resulting orbitals. For a system of three water molecules, we tested this dependence and did not find any significant changes in the occupations of the monomer, samemonomer, and other-monomer part of the individual orbitals. The variation (see Table 1) appears to be in the order of 10 $\mu$-electrons both for the CI-coefficient scaling and the F-matrix mixing. For calculating the variation, we determine first the average population of each occupied orbital, and evaluate the sum of the quadratic deviations from these averages in each calculation. As the calculations converge in quite different numbers of iterations (see Table 1), this should be considered as reasonably stable.

3.2. Shape of the Orbitals. For the functionals LDA, ${ }^{42}$ PW91, ${ }^{43}$ BLYP $^{44}$ and B3LYP ${ }^{45}$ (and Hartree-Fock), we ran the series from two to five water molecules.

We may look (in Table 2) at the most diffuse (and overlapping) orbital at one extremity of our small water chains (the left one in Figure 1). From the five occupied orbitals of the first molecule in our series, this is the highest in orbital energy.

We recognize that in the Hartree-Fock case, the orbitals are slightly less deformed than DFT orbitals and that the different contributions seem stable for the 5-membered chain. The former parallels the usual observation that Hartree-Fock polarizabilities are smaller than DFT polarizabilities. ${ }^{41}$

Note that these orbitals are not Boys or Pipek-Mezey localized orbitals, producing lone-pairs or bond orbitals for instance. The spatial localization appears through the small- 
Table 1. Convergence Acceleration through Cl-Vector Scaling or Fock-Matrix Mixing between Iterations, for three Water Molecules in a standard Van Duijneveldt Basis ${ }^{a}$

\begin{tabular}{|c|c|c|c|c|c|c|}
\hline \multirow[b]{2}{*}{ mixing/scaling } & \multicolumn{6}{|c|}{ Cl-Vector Scaling } \\
\hline & $15 \%$ & $20 \%$ & $25 \%$ & $30 \%$ & $35 \%$ & $40 \%$ \\
\hline no. iterations & 42 & 29 & 25 & 20 & 15 & 23 \\
\hline$\sigma$ (monomer population) $/ 10^{-5}$ & 1.8 & 1.8 & 1.7 & 1.7 & 1.6 & 1.6 \\
\hline$\sigma$ (same orb pop) $/ 10^{-5}$ & 1.6 & 1.5 & 1.5 & 1.5 & 1.4 & 1.4 \\
\hline \multirow[t]{2}{*}{$\sigma$ (other orb pop) $/ 10^{-5}$} & 0.9 & 0.8 & 0.8 & 0.8 & 0.7 & 0.7 \\
\hline & \multicolumn{6}{|c|}{ Fock-Matrix Mixing } \\
\hline mixing/scaling & $75 \%$ & $80 \%$ & $85 \%$ & $90 \%$ & $95 \%$ & \\
\hline no. iterations & 40 & 28 & 35 & 52 & 94 & \\
\hline$\sigma$ (monomer population) $/ 10^{-5}$ & 2.4 & 2.2 & 2.0 & 1.9 & 1.7 & \\
\hline$\sigma$ (same orb pop) $/ 10^{-5}$ & 2.1 & 1.9 & 1.8 & 1.6 & 1.6 & \\
\hline$\sigma$ (other orb pop) $/ 10^{-5}$ & 1.2 & 1.0 & 0.9 & 0.8 & 0.7 & \\
\hline
\end{tabular}

${ }^{a}$ Outside the given range, the calculations did not converge. The next lines give the mean standard deviations of the populations from the average values of the 15 occupied orbitals in the three-molecule cluster. The data is for the BLYP functional.

Table 2. Decomposition of the Most Diffuse Occupied Orbital of One of the Two Extremities of the Water Chains into the Different Populations ${ }^{a}$

\begin{tabular}{|c|c|c|c|c|}
\hline & $\left(\mathrm{H}_{2} \mathrm{O}\right)_{2}$ & $\left(\mathrm{H}_{2} \mathrm{O}\right)_{3}$ & $\left(\mathrm{H}_{2} \mathrm{O}\right)_{4}$ & $\left(\mathrm{H}_{2} \mathrm{O}\right)_{5}$ \\
\hline \multicolumn{5}{|c|}{ monomer orbital } \\
\hline $\mathrm{HF}$ & 0.9964 & 0.9962 & 0.9961 & 0.9961 \\
\hline LDA & 0.9949 & 0.9945 & 0.9944 & 0.9943 \\
\hline PW91 & 0.9949 & 0.9946 & 0.9944 & 0.9944 \\
\hline BLYP & 0.9950 & 0.9946 & 0.9945 & 0.9944 \\
\hline B3LYP & 0.9944 & 0.9941 & 0.9940 & 0.9939 \\
\hline \multicolumn{5}{|c|}{ same fragment } \\
\hline $\mathrm{HF}$ & -0.0002 & -0.0006 & -0.0011 & -0.0012 \\
\hline LDA & -0.0092 & -0.0093 & -0.0099 & -0.0100 \\
\hline PW91 & -0.0067 & -0.0062 & -0.0067 & -0.0067 \\
\hline BLYP & -0.0050 & -0.0044 & -0.0047 & -0.0048 \\
\hline B3LYP & -0.0173 & -0.0166 & -0.0169 & -0.0170 \\
\hline \multicolumn{5}{|c|}{ other fragments } \\
\hline $\mathrm{HF}$ & 0.0038 & 0.0045 & 0.0050 & 0.0052 \\
\hline LDA & 0.0143 & 0.0148 & 0.0155 & 0.0157 \\
\hline PW91 & 0.0117 & 0.0117 & 0.0122 & 0.0124 \\
\hline BLYP & 0.0100 & 0.0098 & 0.0102 & 0.0104 \\
\hline B3LYP & 0.0229 & 0.0225 & 0.0229 & 0.0231 \\
\hline
\end{tabular}

${ }^{a}$ Note that we treat Hartree-Fock on the same footing as the density-functional results.

ness of the contributions $\mathrm{S}$ and $\mathrm{O}$ of eq 7. Small differences are observed between different functionals, reflecting the different importance of self-interaction in the functionals. This leads commonly to the slightly different literature values of, for instance, charge transfer terms ${ }^{41}$ which we may identify as the other-fragment parts in our decomposition. In the case studied, we see that the B3LYP orbital does not present values intermediate between Hartree-Fock and pure exchange-correlation functionals without an explicit exchange term. Already for interaction energies of the three-molecule system B3LYP $(1.15 \mathrm{kcal} / \mathrm{mol})$ is lower than BLYP $(2.86$ $\mathrm{kcal} / \mathrm{mol})$ and Hartree-Fock $(6.12 \mathrm{kcal} / \mathrm{mol})$.

Differently than for the atoms-in-molecules (AIM) approach, ${ }^{46}$ where atomic basins and their populations are studied, we may look at the density attributable to each molecule through the molecular orbitals. This decomposition scheme, based on orbitals rather than on the density, is extensively studied for instance by Fernández Rico et al. ${ }^{47,48}$

3.3. Local Properties As Dipole Moments. Apart from the question of linear scaling methods, we may employ the so-constructed orbitals for studying weakly interacting complexes by extracting monomer properties and interaction-

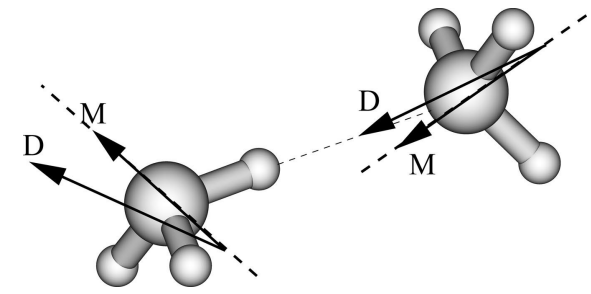

Figure 2. Schematic drawing of the deformation of the molecular dipoles via the interaction of two $\mathrm{NH}_{3}$ molecules. $\mathrm{M}$ indicates the monomer dipoles, and $\mathrm{D}$, the dimer dipoles.

induced changes of these. As an example, we may look at the dipole moment of two interacting $\mathrm{NH}_{3}$ molecules, for which we calculate the influence of the interaction of the size and the direction of the local dipole moments. The global dipole is a well-known quantity, and its measurement leads to an identification of the complex ${ }^{49,50}$ invalidating the common picture of a purely hydrogen-bonded system, with one of the hydrogen atoms lying in the $\mathrm{N}-\mathrm{N}$ connection line. Nevertheless, we use that geometry for the present case, as we are not aiming at a complete study of this dimer system.

For each molecule, we may calculate a dipole moment within the full dimer basis separately and look for the deformation of the monomer-attributed orbitals when constructing the localized dimer orbitals via the described singles-CI procedure. We have a good trace for the deformation, as the iterations only deform in a minimal sense the starting guess orbitals. Figure 2 shows schematically the reorientation of the two moments toward an alignment. This is expected from basic physics for lowering the total energy in a dipole-dipole interaction. However, the contribution of these calculated dipoles to the interaction energy is rather small, being in the order of microhartrees (Table 3 ).

The global moment is varying from about $2 \mathrm{D}$ to about 3 $\mathrm{D}$, much too large compared to the experimental estimate of $0.75 \mathrm{D}^{49}$

3.4. Intermolecular Interactions. Energy decomposition schemes ${ }^{29,51}$ furnish two first-order terms, an electrostatic interaction of the monomers $E_{\mathrm{es}}$ (or $E_{\mathrm{pol}}^{(100)}$ in SAPT) and an exchange-repulsion term $E_{\text {exch-rep }}$ (or Pauli repulsion), the sum of which is called frozen-core contribution $E_{\mathrm{FC}}$ in the CSOV scheme of Bagus and Illas. ${ }^{41,51}$ This $E_{\mathrm{FC}}$ is equivalent 
Table 3. Deformation of the Dipoles of the Individual, Separated Monomers (dipole (M)) toward the Attributed Dipole in the Interacting System (dipole (D)), Calculated with Different Functionals ${ }^{a}$

\begin{tabular}{lcccc}
\hline $\mathrm{NH}_{3}-\mathrm{NH}_{3}$ & dipole (M) & dipole (D) & difference & angle \\
\hline $\mathrm{HF}$ & 1.56 & 1.81 & 0.25 & 15.3 \\
& 1.56 & 1.75 & 0.19 & 0.1
\end{tabular}

\begin{tabular}{|c|c|c|c|c|}
\hline \multicolumn{2}{|c|}{ total dipole (M) } & total dipole (D) & angle (M) & angle (D) \\
\hline \multicolumn{2}{|c|}{2.42} & 3.05 & 77.8 & 62.4 \\
\hline LDA & $\begin{array}{l}1.45 \\
1.44\end{array}$ & $\begin{array}{l}1.66 \\
1.88\end{array}$ & $\begin{array}{l}0.20 \\
0.44\end{array}$ & $\begin{array}{r}13.5 \\
1.2\end{array}$ \\
\hline \multicolumn{2}{|c|}{ total dipole (M) } & total dipole (D) & angle (M) & angle (D) \\
\hline \multicolumn{2}{|c|}{2.25} & 3.02 & 77.8 & 63.0 \\
\hline PW91 & $\begin{array}{l}1.42 \\
1.39\end{array}$ & $\begin{array}{l}1.62 \\
1.77\end{array}$ & $\begin{array}{l}0.20 \\
0.39\end{array}$ & $\begin{array}{r}14.8 \\
1.2\end{array}$ \\
\hline
\end{tabular}

\begin{tabular}{|c|c|c|c|c|}
\hline total & $(\mathrm{M})$ & total dipole (D) & angle (M) & angle (D) \\
\hline \multicolumn{2}{|c|}{2.19} & 2.92 & 77.8 & 61.8 \\
\hline BLYP & 1.40 & 1.62 & 0.211596 & 15.5 \\
\hline
\end{tabular}

\begin{tabular}{|c|c|c|c|}
\hline total dipole (M) & total dipole (D) & angle (M) & angle (D) \\
\hline 2.16 & 2.88 & 77.3 & 60.7 \\
\hline B3LYP & $\begin{array}{l}1.68 \\
1.75\end{array}$ & $\begin{array}{l}0.22 \\
0.32\end{array}$ & $\begin{array}{r}15.0 \\
0.8\end{array}$ \\
\hline
\end{tabular}

\begin{tabular}{cccc}
\hline total dipole $(M)$ & total dipole (D) & angle (M) & angle (D) \\
\hline 2.25 & 2.95 & 77.5 & 61.7
\end{tabular}

\footnotetext{
a The phrase "total dipole" stands for the vector sum of the two individual dipoles, forming the indicated angle. Units are Debye for the dipole moments, and we give differences in length and orientation. The differences for the two monomer dipoles (M) within a calculation has to be ascribed to a non-negligible BSSE, as the basis sets were elaborated for Hartree-Fock orbitals.
}

to the Heitler-London energy, employing the unperturbed monomers orbitals, and corresponds to the antisymmetrized Hartree product of the isolated monomer wave functions.

Orbital relaxation toward the multimer orbitals yields the second-order term called orbital interaction, ${ }^{29,40}$ corresponding to the sum of polarization and charge transfer in CSOV (named induction in the force-field terminology $\mathrm{y}^{33}$ ). In that, scheme relaxation implying virtual orbitals is allowed in this step, in contrast to the first-order, where no mixing between occupied and virtual orbitals is permitted. The subtle question, at which instance a correction for a basis-set superposition error (BSSE) has to be included, is circumvented in our scheme as we calculate monomers in the monomer atomic basis and as well in the multimer atomic basis, and both can be compared to the final HF or DFT solution. Thus, results can be correlated to schemes respecting the BSSE in the very last step like CSOV or to schemes calculating intermolecular interactions in the full multimo- lecular basis sets throughout like SAPT. The EDA approaches decompose the total interaction energy $\Delta E$ as

$$
\Delta E=E_{\mathrm{es}}+E_{\mathrm{exch}-\mathrm{rep}}+E_{\mathrm{OI}}+E_{\mathrm{BSSE}}=E_{\mathrm{FC}}+E_{\mathrm{OI}}+E_{\mathrm{BSSE}}
$$

with the terms defined above.

CSOV (using canonical orbitals) was used to provide accurate data for the development of a new generation of force fields based on the electron density. ${ }^{14}$ The present approach in fragment-localized molecular orbitals permits to find corresponding terms, which will be detailed in a forthcoming paper. Here, we limit ourselves to the global terms discussed above (details about equivalence of terms can be found in refs 40 and 41).

We tested the accuracy of our approach on a previously investigated linear water dimer configuration. ${ }^{14,31,32}$ As expected, we observe the invariance to orbitals localization of our decompositions scheme: localized and canonical orbitals lead to rigorously the same energy contributions, which is not the case for all decomposition schemes (due to projections or approximative orbital rotations).

Table 4 displays the comparison between our approach (fragment-localized orbitals) and CSOV (canonical orbitals) for different functionals, including Hartree-Fock. Differences between the two approaches appear to be around 0.1 $\mathrm{kcal} / \mathrm{mol}$ and are due to a slightly smaller variational space, $6 \mathrm{~d}$ or $5 \mathrm{~d}$ orbitals in the $\mathrm{AO}$ basis, and different integration grids The already observed (refs 41 and 52) DFT exchangerepulsion-functional-specific behavior is observed. For example, PW91 exhibits a less repulsive value of the exchangerepulsion contribution compared to HF.

Table 4 shows as well the importance of taking into account the full dimer basis. Indeed the addition of the second monomer basis functions clearly affects the energy components by augmenting both the value of electrostatic and the exchange-repulsion energy. This "BSSE-like" effect is then clearly pronounced for frozen core (or first order in the SAPT terminology). Such a behavior, already observed (see refs 32 and 53) in the framework of SAPT should be carefully taken into account when developing force fields with a precision within this order of magnitude.

The decompositions may be easily extended beyond dimer systems, allowing the calculation of many-body contributions in contrast to SAPT or CSOV calculations, often restricted to the implementation of 2-body terms. ${ }^{14}$ Another multimonomer scheme, the reduced variational space scheme ${ }^{54}$ (similar to CSOV) was applied for Hartree-Fock wave functions and may be extended easily to Kohn-Sham wave functions as well.

As it can be seen, once again the specific behavior of each functional for the exchange-repulsion component is confirmed: PW91 < B3LYP < HF < BLYP.

Table 5 gathers results only for the intermolecular contributions on three and five water-molecule chains. Such water chains were initially selected in order to enhance cooperative effects as the molecular dipole moments are parallel to the main axis of the helixlike chain. ${ }^{33}$

Such chains also confirmed the presence of an enhanced charge transfer at the DFT level since $E_{\mathrm{OI}}$ is strongly 
Table 4. Energy Decomposition $(\mathrm{kcal} / \mathrm{mol})$ for the Linear Water Dimer ${ }^{a}$

\begin{tabular}{|c|c|c|c|c|c|}
\hline functional & $E_{\mathrm{es}}$ & $E_{\text {exch-rep }}$ & $E_{\mathrm{FC}}$ & $E_{\mathrm{OI}+\mathrm{BSSE}}$ & total interaction \\
\hline \multicolumn{6}{|c|}{ monomers in the respective monomer basis } \\
\hline $\mathrm{HF}(5 \mathrm{D})$ & -8.26 & 6.88 & -1.38 & -2.16 & -3.55 \\
\hline $\mathrm{HF}(6 \mathrm{D})$ & -8.27 & 6.91 & $-1.36(-1.37)$ & $-2.18(-2.19)$ & -3.55 \\
\hline BLYP & -8.00 & 7.42 & -0.58 & -3.28 & -3.86 \\
\hline BLYP (6D) & -8.03 & 7.48 & $-0.55(-0.61)$ & $-3.30(-3.26)$ & -3.86 \\
\hline PW91 & -7.91 & 6.22 & -1.69 & -3.30 & -4.99 \\
\hline PW91 (6D) & -7.93 & 6.27 & $-1.66(-1.50)$ & $-3.33(-3.48)$ & -4.99 \\
\hline B3LYP (6D) & -8.07 & 6.71 & $-1.36(-1.45)$ & $-2.99(-2.95)$ & $-4.35(4.40)$ \\
\hline \multicolumn{6}{|c|}{ monomers in the dimer basis } \\
\hline $\mathrm{HF}(5 \mathrm{D})$ & -8.30 & 7.02 & -1.28 & -2.27 & -3.55 \\
\hline $\mathrm{HF}(6 \mathrm{D})$ & -8.30 & 7.02 & -1.28 & -2.27 & -3.55 \\
\hline BLYP & -8.19 & 7.68 & -0.51 & -3.35 & -3.86 \\
\hline PW91 & -8.10 & 6.52 & -1.58 & -3.42 & -4.99 \\
\hline B3LYP & -8.18 & 6.87 & -1.31 & -3.04 & -4.35 \\
\hline
\end{tabular}

${ }^{a}$ Geometry and basis set are from ref 59. Results from CSOV are given in parenthesis. The Hartree-Fock results are given for a 5D and $6 \mathrm{D}$ AO basis set as CSOV is done in the latter. In the DFT case, our scheme uses 5D throughout. The effect on the different terms is small. For the second part taking $6 \mathrm{D}$ or $5 \mathrm{D}$ functions makes no difference for all functionals.

Table 5. Energy Decomposition Results $(\mathrm{kcal} / \mathrm{mol})$ for Three and Five Water Molecules in the Helix Structure ${ }^{a}$

\begin{tabular}{|c|c|c|c|c|c|c|}
\hline & functional & $E_{\mathrm{es}}$ & $E_{\text {exch-rep }}$ & $E_{\mathrm{OI}}$ & BSSE & total interaction \\
\hline \multirow[t]{5}{*}{ three water molecules } & $\mathrm{HF}$ & -39.33 & 66.14 & -21.19 & 0.51 & 6.12 \\
\hline & BLYP & -40.85 & 69.51 & -26.88 & 1.08 & 2.86 \\
\hline & PW91 & -40.03 & 64.49 & -27.15 & 1.07 & -1.62 \\
\hline & B3LYP & -40.24 & 65.82 & -25.29 & 0.89 & 1.15 \\
\hline & $\mathrm{HF}$ & -80.88 & 132.08 & -45.33 & 1.03 & 6.87 \\
\hline \multirow[t]{2}{*}{ five water molecules } & BLYP & -83.51 & 138.89 & -57.16 & 2.19 & 0.41 \\
\hline & PW91 & -81.86 & 128.74 & -57.75 & 2.16 & -8.71 \\
\hline
\end{tabular}

${ }^{a}$ Note the strongly different results of the total interaction energy for the different functionals.

increased in agreement with previous studies. ${ }^{41}$ The (fragment-)localized approach offers as well an energy decomposition per orbital or molecule beyond the global terms.

\section{Conclusion}

We showed that the singles-CI scheme used for the construction of localized Hartree-Fock orbitals applies as well for the construction of (fragment-)localized Kohn-Sham orbitals. Through the use of canonical monomer orbitals, the localized orbitals of the whole, fragment-based system are very well defined and reproducible, even if an explicit localization criterion is absent in the procedure. Cutting the orbitals in three parts permits to decompose monoelectronic properties in a straightforward manner into monomer, polarization, and charge-transfer parts. This decomposition based on orbital contributions is, as any decomposition scheme of global observables, somehow arbitrary.

For the moment, the implementation gives the exact equivalence to canonical orbitals, but cutoffs, expansion of $\mathbf{S}^{-1 / 2}$ into a power series, and other selection criteria may be implemented easily, leading to a potentially linear-scaling procedure.

We do not doubt that the monomer-attributed properties (as dipole moments in the present case) will show useful for interpretation and the construction of modern force fields.

Acknowledgment. One of the authors (P.R.) wishes to express his gratitude to the teaching of the outlined methods for generating localized Hartree-Fock orbitals by J.-P. Malrieu and Jean-Pierre Daudey in Toulouse. The work of R. Lefebvre has been brought to the attention of the authors by G. Berthier (Paris). The work has been financed via the French government (University of Paris VI) and the CNRS. Additional help from the ACI Simulation in Molecules (C. Le Bris, CERMICS) and from the French ANR (Agence national pour la recherche), project WADEMECOM (contract No ANR-07-BLAN-0271) was very welcome. The CSOV calculations were made possible by the CINES (Montpellier, France).

Note Added in Proof. During the processing of the manuscript, we had to learn the sad news that Jean-Pierre Daudey passed away in September 2008, without being able to see the final article.

\section{Appendix A: Technical Details}

For the calculations, we use our series of experimental programs,${ }^{55}$ which are linked to DALTON, ${ }^{56}$ for the generation of integrals, and to Molpro, ${ }^{57}$ for the evaluation of the density functional terms. For the CSOV calculation we employed an in-house implementation ${ }^{41}$ of the method in HONDO 95.3. ${ }^{58}$

The basis set for the water molecules is a standard one of van Duijneveldt, ${ }^{59}$ designed explicitly for the computation of intermolecular interactions in water aggregates. ${ }^{60}$ The individual water molecules are in their ground-state geometry ${ }^{31,32}$ and are assembled in a helixlike linear arrangement.

For the ammonia molecules, an augmented v. Duijneveldt basis and standard geometry are employed, in the same line as for our previous studies (e.g., ref 40). 
Calculations were carried out on a Pentium IV-based personal computer (local DFT) and on SP5 IBM processors (CSOV).

\section{Appendix B: Equivalence of HF and DFT Singles-CI Procedure}

The fact that the construction of Hartree-Fock orbitals through the singles-CI method is useful has been demonstrated several times since its proposition, in particular for periodic systems. ${ }^{19,37,61}$ The derivation of the singles-CI equations through the minimization of the Rayleigh quotient with respect to the expansion coefficients in the space of determinant may be found in standard textbooks. ${ }^{62}$

What remains in this section is to show that the minimization of the DFT energy functional with respect to a multideterminantal wave function leads to similar expressions for the matrix elements of the CI matrix. It is of little importance whether we start with determinants or simple Hartree products of orbitals - the resulting densities are the same. For the sake of similarity to the derivation of the usual CI equations, we stick to determinants and the common Slater rules.

Our ansatz for the wave function as linear combination of singly excited determinants leads directly to a CI density as function of three-dimensional coordinates

$$
\begin{gathered}
\psi=c_{0 \Phi 0}+\sum_{I} c_{I \Phi I} \\
\rho(\vec{r})=N \int \cdots \int \mathrm{d}^{3} r_{1} \cdots \mathrm{d}^{3} r_{N-1} \psi^{\dagger}\left(\vec{r}_{1}, \cdots, \vec{r}_{N-1}, \vec{r}\right) \times \\
\psi\left(\vec{r}_{1}, \cdots, \vec{r}_{N-1}, \vec{r}\right)=c_{0}^{2} \rho_{\Phi_{0}}(\vec{r})+ \\
\sum_{I} c_{I}^{2} \rho_{\Phi_{I}}(\vec{r})+2 N \sum_{I<J} c_{I} c_{J} \int \cdots \int \mathrm{d}^{3} r_{1} \cdots \\
\mathrm{d}^{3} r_{N-1} \Phi_{I}^{\dagger}\left(\vec{r}_{1}, \cdots, \vec{r}_{N-1}, \vec{r}\right) \Phi_{J}\left(\vec{r}_{1}, \cdots, \vec{r}_{N-1}, \vec{r}\right)= \\
c_{0}^{2} \rho_{\Phi_{0}}(\vec{r})+\sum_{I} c_{I}^{2} \rho_{\Phi_{I}}(\vec{r})+2 \sum_{I<J} c_{I} c_{J} \phi_{k}^{I}(\vec{r}) \phi_{l}^{J}(\vec{r})
\end{gathered}
$$

where $k$ and $l$ are the number of the single orbital by which the two determinants $\Phi_{I}$ and $\Phi_{J}$ differ (in the double sum $I$ $<J$, we allow $\Phi_{I}=\Phi_{0}$ ). If there are more orbital differences, no contribution to the density is obtained, as the density operator is a monoelectronic operator. In a second quantization, we may write the density associated with the two determinants as

$$
\rho_{I J}(\vec{r})=N \phi_{k}(\vec{r}) \phi_{l}(\vec{r})\left\langle\Phi_{I}\left|a_{k}^{\dagger} a_{l}\right| \Phi_{J}\right\rangle
$$

as the (N-1)-particle determinants $a_{l}\left|\Phi_{J}\right\rangle$ and $a_{k}\left|\Phi_{I}\right\rangle$ need to be equal to yield an integral different from zero.

With this expression at hand, we may write up the variation of the density-dependent exchange-correlation functional. ${ }^{63,64}$ With the formal matrix element of the exchange-correlation potential $v^{\mathrm{XC}}(\vec{r})$ as a one-electron operator $V^{\mathrm{XC}}=\Sigma_{i} v^{\mathrm{XC}}\left(\vec{r}_{i}\right)$ acting on determinants, we have

$$
\int v^{\mathrm{XC}}(r) \rho_{I}(\vec{r}) \mathrm{d}^{3} r=\left\langle\Phi_{I}\left|V^{\mathrm{XC}}\right| \Phi_{I}\right\rangle
$$

and

$$
\int v^{\mathrm{XC}}(r) \phi_{K}^{I}(\vec{r}) \phi_{l}^{J}(\vec{r}) \mathrm{d}^{3} r=\left\langle\Phi_{I}\left|V^{\mathrm{XC}}\right| \Phi_{J}\right\rangle
$$

and thus using

$$
\frac{\partial \rho(\vec{r})}{\partial c_{I}}=2 c_{I} \rho_{\Phi_{I}}(\vec{r})+2 \sum_{J \neq I} c_{J} \phi_{k}^{I}(\vec{r}) \phi_{l}^{J}(\vec{r})
$$

we obtain

$$
\begin{aligned}
& \frac{\delta E^{X C}[\rho]}{\delta c_{I}}=\left.\int \underbrace{\frac{\delta E^{X C}[\rho]}{\delta \rho}}_{\nu^{X C}(\overrightarrow{\mathrm{r}})}\right|_{\vec{r}} \frac{\partial \rho(\overrightarrow{\mathrm{r}})}{\partial c_{l}} d^{3} r \\
& =2 c_{I}\left\langle\boldsymbol{\Phi}_{I}\left|V^{X C}\right| \boldsymbol{\Phi}_{I}\right\rangle+2 \sum_{I \neq J} c_{J}\left\langle\boldsymbol{\Phi}_{I}\left|V^{X C^{X}}\right| \boldsymbol{\Phi}_{J}\right\rangle
\end{aligned}
$$

For the Coulomb matrix elements, the same construction holds: again with a one-electron operator $\mathbf{J}$. All terms of the

$$
\begin{aligned}
& E_{J}[\rho]=\frac{1}{2} \iint \frac{\rho\left(\overrightarrow{\mathrm{r}}_{1}\right) \rho\left(\overrightarrow{\mathrm{r}}_{2}\right)}{\left|\overrightarrow{\mathrm{r}}_{1}-\overrightarrow{\mathrm{r}}_{2}\right|} d^{3} r_{1} d^{3} r_{2} \\
& \frac{\delta E_{J}}{\delta c_{I}}=\int \underbrace{\left.\frac{\delta E_{J}[\rho]}{\delta \rho}\right|_{\vec{r}}} \quad \frac{\partial \rho(\overrightarrow{\mathrm{r}})}{\partial c_{I}} d^{3} r
\end{aligned}
$$

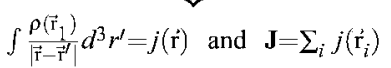

$$
\begin{aligned}
& \frac{\delta E_{J}[\rho]}{\delta c_{I}}=2 c_{I}\left\langle\Phi_{I}|\mathbf{J}| \Phi_{I}\right\rangle+2 \sum_{I \neq J} c_{J}\left\langle\Phi_{I}|\mathbf{J}| \Phi_{J}\right\rangle
\end{aligned}
$$

DFT energy functional put together, we arrive at the same $\mathrm{CI}$ equations as for a standard Hartree-Fock case, with the monoelectronic Kohn-Sham matrix $\mathscr{K}=T+Z+J+$ $V^{\mathrm{XC}}$ instead of the Hamilton operator $H=T+Z+1 / r_{12}$ (with kinetic energy $T$ and electron-nucleus attraction $Z$ ):

$$
\begin{aligned}
& E=\left\langle\Phi_{0}|\mathscr{K}| \Phi_{0}\right\rangle+\sum_{I} c_{I}\left\langle\Phi_{0}|\mathscr{K}| \Phi_{I}\right\rangle \\
& c_{I} E=\left\langle\Phi_{0}|\mathscr{K}| \Phi_{I}\right\rangle+c_{I}\left\langle\Phi_{I}|\mathscr{K}| \Phi_{I}\right\rangle+\sum_{J \neq 0, I} c_{J}\left\langle\Phi_{I}|\mathscr{K}| \Phi_{J}\right\rangle
\end{aligned}
$$

As such, only determinants with one difference in the orbital occupation interact, and no approximation through the neglect of pure bielectronic integrals as for Hamilton matrix elements is necessary.

Thus, in the end exactly the same optimization scheme for the self-consistent orbitals may be employed, for Hartree-Fock or Kohn-Sham orbitals, including hybrid functionals with a nonzero exact-exchange term. This is made possible by the neglect of the pure bielectronic integrals, of which the exchange part is replaced in Kohn-Sham theory by the monoelectronic Kohn-Sham potentials.

\section{References}

(1) Ochsenfeld, C.; Kussmann, J.; Lambrecht, D. S. Linear-Scaling Methods in Quantum Chemistry. Reviews in Computational Chemistry; Lipkowitz, K. B., Cundari, T. R., Eds.; WileyVCH: New York, 2007; Vol. 23, p 1.

(2) Maslen, P. E.; Ochsenfeld, C.; White, C. A.; Lee, M. S.; HeadGordon, M. J. Phys. Chem. A 1998, 102, 2215.

(3) Boys, S. F. Rev. Mod. Phys. 1960, 32, 296.

(4) Edmiston, C.; Ruedenberg, K. Rev. Mod. Phys. 1963, 34, 457.

(5) Pipek, J.; Mezey, P. J. Chem. Phys. 1989, 90, 4916.

(6) Foster, J. P.; Weinhold, F. J. Am. Chem. Soc. 1980, 102, 7211.

(7) Reed, A. E.; Weinhold, F. J. Chem. Phys. 1985, 83, 1736. 
(8) Marzari, N.; Vanderbilt, D. Phys. Rev. B 1997, 56, 12847.

(9) Rob, F.; Podeszwa, R.; Szalewicz, K. Chem. Phys. Lett. 2007, $445,315$.

(10) Cisneros, G. A.; Piquemal, J.-P.; Darden, T. A. J. Chem. Phys. 2005, 123, 044109.

(11) Gresh, N.; Cisneros, G. A.; Darden, T. A.; Piquemal, J.-P. J. Chem. Theo. Comput. 2007, 3, 1960.

(12) Khaliullin, R. Z.; Bell, A. T.; Head-Gordon, M. J. Chem. Phys. 2006, 124, 204105.

(13) Khaliullin, R. Z.; Cobar, E. A.; Lochan, R. C.; Bell, A. T.; Head-Gordon, M. J. Phys. Chem. A 2007, 111, 8753.

(14) Piquemal, J.-P.; Cisneros, G. A.; Reinhardt, P.; Gresh, N.; Darden, T. A. J. Chem. Phys. 2006, 124, 104101.

(15) The idea had been introduced and applied to model Hamiltonians in order to devise a general scheme for obtaining a solution to the closed and open-shell Hartree-Fock equations in a finite basis well before. (a) Lefebvre, R.; Moser, C. M. J. Chim. Phys. (in French) 1956, 53, 393. (b) Lefebvre, R. Une méthode de détermination des orbitales moléculaires selfconsistentes. Thesis, University of Paris, 1959 (in french). (c) Lefebvre, R. C.R. Acad. Sci. 1955, 240, 1094.

(16) Daudey, J.-P. Chem. Phys. Lett. 1974, 24, 574.

(17) Gineityte, V. J. Mol. Struc-THEOCHEM 2007, 810, 91.

(18) Maynau, D.; Evangelisti, S.; Guihery, N.; Calzado, C. J.; Malrieu, J.-P. J. Chem. Phys. 2002, 116, 10060.

(19) Reinhardt, P.; Malrieu, J.-P.; Povill, A.; Rubio, J Int. J. Quantum Chem. 1997, 70, 167.

(20) Ángyán, J. G.; Gerber, I. C.; Savin, A. Toulouse J. Phys. Rev. A 2005, 72, 12510.

(21) Koch, W.; Holthausen, M. C. A Chemist's Guide to Density Functional Theory, 2nd ed.; Wiley-VCH: Weinheim, Germany, 2002.

(22) Heßelmann, A.; Jansen, G. Chem. Phys. Lett. 2002, 357, 464.

(23) Misquitta, A. J.; Podeszwa, B.; Jeziorski, K.; Szalewicz, J. Chem. Phys. 2005, 123, 214103.

(24) Adams, W. H. J. Chem. Phys. 1961, 34, 89.

(25) Gilbert, T. L. J. Chem. Phys. 1974, 60, 3835.

(26) Kunz, A. B. Theor. Chem. Acta 1993, 84, 353.

(27) Seijo, L.; Barandiaran, Z. Int. J. Quantum Chem. 1996, 60, 617.

(28) Ziegler, T.; Rauk, A. Inorg. Chem. 1979, 18, 1755.

(29) Bickelhaupt, F. M.; Baerends, E. J. In Reviews in Computational Chemistry; Lipkowitz, K. B., Boyd, D. B., Eds.: Wiley-VCH: New York, 2000; Vol. 15, pp 1-86.

(30) Bagus, P. S.; Herrmann, K.; Bauschlicher, C. W. J. Chem. Phys. 1984, 80, 4378.

(31) Tschumper, G. S.; Leininger, M. L.; Hoffman, B. C.; Valeev, E. F.; Quack, M.; Schaefer, H. F., III J. Chem. Phys. 2002, 116,690

(32) van Duijneveldt-van de Rijdt, J. G. C. M.; Mooij, W. T. M.; van Duijneveldt, F. B. Phys. Chem. Chem. Phys. 2003, 5, 1169.

(33) Piquemal, J.-P.; Chelli, R.; Procacci, P.; Gresh, N. J. Phys. Chem. A 2007, 111, 8170.

(34) Löwdin, P. O. J. Chem. Phys. 1950, 18, 365.

(35) Aiken, J. G.; Erdos, J. A.; Goldstein, J. A. Int. J. Quantum Chem. 1980, 18, 1101.
(36) Daudey, J.-P.; Heully, J.-L.; Malrieu, J.-P. J. Chem. Phys. 1993, 99, 1240.

(37) Rubio, J.; Povill, A.; Malrieu, J.-P.; Reinhardt, P. J. Chem. Phys. 1997, 107, 10044.

(38) Morokuma, K. J. Chem. Phys. 1971, 55, 1236.

(39) Kitaura, K.; Morokuma, K. Int. J. Quantum Chem. 1976, 10, 325.

(40) Langlet, J.; Bergès, J.; Reinhardt, P. J. Mol. Struct.THEOCHEM 2004, 685, 43.

(41) Piquemal, J.-P.; Marquez, A.; Parisel, O.; Giessner-Prettre, C. J. Comput. Chem. 2005, 26, 1052.

(42) Vosko, S. H.; Wilk, L.; Nusair, M. Can. J. Phys. 1980, 58, 1200.

(43) Perdew, J. P.; Wang, Y. Phys. Rev. B 1992, 45, 13244.

(44) Lee, C. L.; Yang, W.; Parr, R. G. Phys. Rev. B 1988, 37, 785.

(45) Stephens, P. J.; Devlin, F. J.; Chabalowski, C. F.; Frisch, M. J. J. Phys. Chem. 1994, 98, 11623.

(46) Bader, R. F. W. In Atoms in Molecules: A Quantum Theory; Oxford Univ. Press: Oxford, Great Britain, 1990.

(47) Fernández Rico, J.; López, R.; Ema, I.; Ramíre, G. J. Comput. Chem. 2004, 25, 1347.

(48) Fernández Rico, J.; López, R.; Ramírez, G.; Ema, I.; Ludena, E. V. J. Comput. Chem. 2004, 25, 1355.

(49) Nelson, D. D.; Klemperer, W.; Fraser, G. T.; Lovas, F. J.; Suenram, R. D. J. Chem. Phys. 1985, 87, 6364.

(50) Lee, J. S.; Park, S. Y. J. Chem. Phys. 2000, 112, 230.

(51) Bagus, P. S.; Illas, F. J. Chem. Phys. 1992, 96, 8962.

(52) Langlet, J.; Bergès, J.; Reinhardt, P. Chem. Phys. Lett. 2004, 396, 10.

(53) van Duijneveldt-van der Rijdt, J. G. C. M.; van Duijneveldt, F. B. J. Chem. Phys. 1992, 97, 5019.

(54) Stevens, W. J.; Fink, W. H. Chem. Phys. Lett. 1987, 139, 15.

(55) Reinhardt, P. ORTHO, Different Programs to Perform Abinitio Calculations within Highly Localized Orbitals, unpublished, 1998-2008.

(56) Helgaker, T.; Jensen, H. J. Aa.; Jørgensen, P.; Olsen, J.; Ruud, K.; Ågren, H.; Andersen, T.; Bak, K. L.; Bakken, V.; Christiansen, O.; Dahle, P.; Dalskov, E. K.; Enevoldsen, T.; Fernandez, B.; Heiberg, H.; Hettema, H.; Jonsson, D.; Kirpekar, S.; Kobayashi, R.; Koch, H.; Mikkelsen, K. V.; Norman, P.; Packer, M. J.; Saue, T.; Taylor, P. R.; Vahtras, O. DALTON, version 1.0; University of Oslo, Norway, 1997.

(57) Werner, H. J.; Knowles, P. J. with contributions from Almlöf, J.; Amos, R. D.; Bernhardsson A.; Berning, A.; Cooper, D. L.; Deegan, M. J. O.; Dobbyn, A. J.; Eckert, F.; Hampel, C.; Lindh, R.; Lloyd, A. W.; Meyer, W.; Mura, M. E.; Nicklass, A.; Peterson, K.; Pitzer, R.; Pulay, P.; Rauhut, G.; Schütz, M.; Stoll, H.; Stone, A. J.; Taylor, P. R.; Thorsteinsson, T. Molpro, version 2002.7; University of Stuttgart and Birmingham: Germany and Great Britain, 2003.

(58) Dupuis, M.; Marquez, A.; Davidson, E. R. HONDO95.3; Quantum Chemistry Program Exchange (QCPE), Indiana University: Bloomington, IN, 1995.

(59) van Duijneveldt, F. B. Gaussian basis sets for the atoms $\mathrm{H}$-Ne for use in molecular calculations; IBM Technical Research Report No. RJ-945, IBM: San José, CA, 1971. 
Kohn-Sham Orbitals via a Singles-CI Procedure

(60) Mooij, W. T. M.; van Duijneveldt, F. B.; van Duijneveldtv.d.Rijdt, J. G. C. M.; van Eijck, B. P. J. Phys.Chem. A 1999, 103, 9872.

(61) Sano, T.; Matsuoka, O. Bull. Chem. Soc. Jpn. 1996, 69, 2195.

(62) Szabo, A.; Ostlund, N. In Modern Quantum Chemistry: Introduction to Advanced Electronic Structure Theory; MacMillan Publishing Co.: New York, 1982; p 33.
J. Chem. Theory Comput., Vol. 4, No. 12, 20082029

(63) Pople, J. A.; Gill, P. M. W.; Johnson, B. G. Chem. Phys. Lett. 1992, 199, 557.

(64) Parr, R. G.; Yang, W. In Density-functional theory of atoms and molecules; Oxford University Press: Oxford, Great Britain, 1989.

CT800242N 